\title{
One-pot Synthesis of Dihydroorotate by Multienzyme Polypeptide CAD
}

\author{
Jiang Jiawen \\ Key Laboratory for Food Science and Engineering \\ Harbin Uninversity of Commerce \\ Harbin, China \\ jiangjiawen@163.com \\ Sun Lirui \\ Key Laboratory for Food Science and Engineering \\ Harbin Uninversity of Commerce \\ Harbin, China \\ fere_sun@163.com
}

\author{
Zhu Limin \\ Laboratory of Industrial Biotechnology \\ Donghua University \\ lzhu@dhu.edu.cn
}

\author{
Xin Jiaying* \\ Key Laboratory for Food Science and Engineering \\ Harbin Uninversity of Commerce \\ Harbin, China \\ xinjiayingvip@163.com \\ Corresponding Author
}

\begin{abstract}
In the present research work, the multi-enzyme polypeptide carbamoyl synthetase-aspartate transcarbamoylase- dihydroorotase (CAD) was used as a novel biocatalyst to catalyze a multi-step reaction in a unitary reaction medium. Using organic solvent as reaction medium, we have observed formation of the final product, dihydroorotate, from initial substrates. Study on substrate specificity showed that the enzyme is capable of converting various amine to the corresponding dihydroortate (DHO). The multienzyme polypeptide CAD has an apparent molecular weight of $215,000-222,000$.
\end{abstract}

KeyWords- multienzyme polypeptide; CAD; multi-step reaction; dihydroorotate

\section{INTRODUCTION}

To date, a few complex compounds are synthesized by more than two steps enzymatic catalyzed reaction. In common manner, one enzyme can only catalyzes one-step reaction, which limited consumedly the application of enzyme in complex compounds. System designed with multiple enzymes co-presence in the same reaction medium as one pot biocatalyst is one strategy. However, it is very difficult to control the mult-istep reaction in a given reaction sequence. In addition, intermediates of the pathway may be used in other reactions, diluted, and exposed to bulk solvent where they may be degraded.

Systems with multiple enzymes co-immobilized on the same support is another strategy which have been designed based on the assumption that the advantages associated with natural existing multienzyme complexes i.e., close enzyme proximity, short substrate transient times will also apply to those immobilized system [1]. However, there are several problems to be considered in the case of multiple enzymes co-immobilization. The amounts and ratios of the individual enzymes in a multi-enzyme system, for example, can be varied and should ideally be adjusted so as to achieve an optimal overall rate. To minimize transient times, enzymes catalyzing successive steps in such a process should also be arranged so as to be in close proximity to each other [1]. These goals are difficult to achieve due to the relatively low and unpredictable efficiencies of most procedures for the immobilization of enzymes. On the other hand, when the number of enzymes acting in series more than two, it is very difficult to fix deliberately one enzyme in the vicinity of other enzymes that participate in a given reaction sequence.

Many metabolic reactions in cells are catalyzed by multi-enzyme complexes. For example, fatty acid synthesis, de novo purine biosynthesis, and de novo pyrimidine biosynthesis. The structure of a multienzymatic protein is like a string of beads: a contiguous stretch of polypeptide folds into a domain with a single enzymatic activity and is separated from the next enzymatic domain by a polypeptide " bridge" or "linker". The product of one enzyme is the substrate of the next in such complexes and is channeled directly from one to the other without diffusion from the complex. The multifunctional enzyme can facilitate the direct movement of products of one active site to a nearby catalytic site where it will be a substrate. This greatly increases the rate of flux through the pathway because this allows channeling of the substrate much like that discussed previously with tryptophan synthase. Secondly, intermediates of the pathway are sequestered and not used in other reactions, not diluted, and not exposed to bulk solvent where they may be degraded

The Pyrimidine biosynthetic pathway provides a remarkable example of the use of multifunctional enzymes in metabolism. In mammals and higher eukaryotes, the first three reactions of the pyrimidine pathway are catalyzed by a single polypeptide bearing three active sites. This is a trifunctional enzyme referred to as carbamoyl synthetase-aspartate transcarbamoylase- dihydroorotase (CAD). CAD catalyzes the following reactions, which are carried out by three separate bacterial enzymes: (1) carbamoyl synthetase, (2) aspartate transcarbamoylase, and (3) dihydroorotase. The reactions catalyzed by the CAD are illustrated in Figure 1.[2-6]

The reaction catalyzed by dihydroorotase is reversible and strongly favors the formation of carbamyl aspartate. At 
$\mathrm{pH} 8.0$, the equilibrium ratio of dihydroorotate to carbamyl aspartate is 0.015 .

In this paper, a new strategy to use the multienzyme polypeptide CAD as one pot biocatalyst was developed. The multifunctional polypeptide CAD was used as a novel biocatalyst to catalyze a multi-step reaction in a unitary reaction medium. Because the third reaction catalyzed by dihydroorotase is reversible which unfavours the accumulation of dihydroorotase, non-aqueous organic was used to overcome the unfavorable reaction equilibrium. Study on substrate specificity showed that the enzyme is capable of converting various amino to the corresponding dho, DHO and it derivatives were synthesized from asparate, $\mathrm{HCO}_{3}^{-}$、 and $\mathrm{NH}_{3}$ (or various amine) along with ATP, respectively, confirmed that the substrate channeling in the CAD is big enough to admit intermediates derivatives run through it.

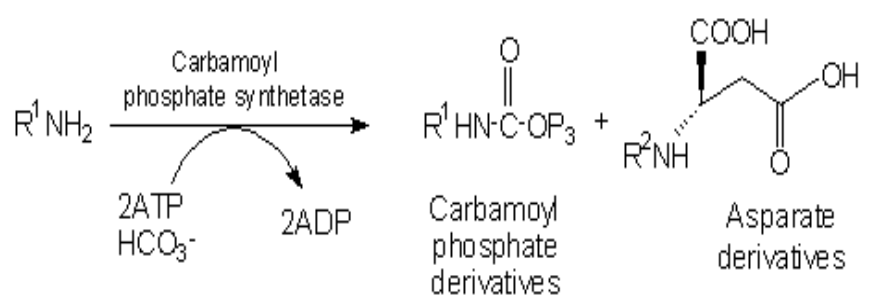

$$
\begin{aligned}
& R^{1}, R^{2} \text { (could ary group } \\
& \text { including chiral groups) }
\end{aligned}
$$

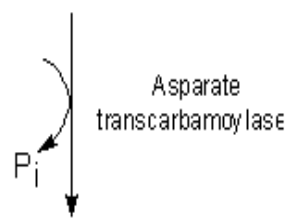<smiles>[R]NC(=O)CC(NC([R])=O)C(=O)O</smiles>

Dihydroortate
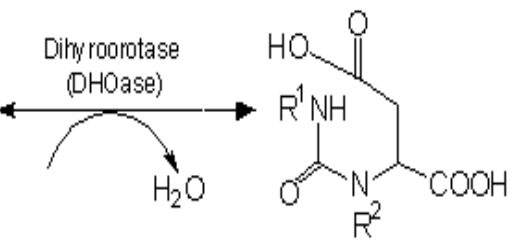

Carbamoyl

aspartate derivatives
Figure 1 . The reactions catalyzed by the CAD multienzyme system ${ }^{[6]}$

\section{MATERIALS AND METHODS}

A. Simultaneous separation by High-performance liquid chromatography (HPLC) of products and substrates

The HPLC conditions provided basedline separation of the DHO AND CA, ATP and ADP. Anion-exchange HPLC system for the separation of ATP, ADP, and dihydroorotate (DHO) was developed. Organic phase containing reaction substrates and products was injected onto a Zarbox column $(150 \mathrm{~mm} \times 4.6 \mathrm{~mm}$ I.D.). Reaction substrates and products were eluted using a linear gradient (buffer A, $0.01 \mathrm{~mol} / \mathrm{L}$ potassium dihydrogen phosphate buffer; buffer $\mathrm{B}, \quad 0.5 \mathrm{~mol} / \mathrm{L}$ potassium dihydrogen phosphate buffer, initial $\mathrm{pH} 4.7$ and 4.6, respectively) and a flow rate of $0.7 \mathrm{ml} / \mathrm{min}$. Compounds were identified by at least two of the following methods: comparing the elution patterns of unknowns with the elution patterns of standards, thin layer chromatography in comparison with standards, or by the spectra for DHO. All products show similar spectra. The absorption spectrum of DHO measured showed no peak over the wave length range $300-240 \mathrm{~nm}$. The spectra shown for the DHO are consistent with the view that the compound exists in the reaction solution. The elution time of these compounds are being used as a method for their estimation.

This HPLC method, enabling simultaneous separation of intermediates in the reaction may be use as a method for their estimation.

Further confirmation of the identity of the compound as DHO was obtained by injectic standard DHO onto the anion-exchange HPLC system. standard DHO eluted at approximately min, i.e. with the same tR value.

Again the products eluted in a peak with the tR value anticipated for a pure standard of DHO, suggesting this products was indeed DHO.

\section{B. Method for extraction and purification of CAD from cells}

Adapted from Coleman et al [2], the multi-enzyme polypeptide CAD was extracted and purified from an overproducing BHK cells. The ammonium sulfate fraction was further purified by DEAE-Sephorase Cl-6B ionic exchange chromatography. The purified protein migrated as a single band of molecular weight $215,000-222,000$ on sodium dodecyl sulfate polyacrylamide gels. We have verified, by sodium dodecyl sulfate polyacrylamide gels electrophoresis, that our preparation of the protein is homogeneous. The protein was stored in a buffer consisting of $20 \mathrm{mM}$ Tris- $\mathrm{HCl}, \mathrm{pH} 7.4,50 \mathrm{mM} \mathrm{KCl}, 25$ $\mathrm{mM} \mathrm{MgCl2} \mathrm{,} \mathrm{4mM} \mathrm{glucamine,} 4 \mathrm{mM}$ aspartate, $0.1 \mathrm{mM}$ EDTA, 1mM dithiothreitol, 30\% (V/V) dimethyl sulfoxide and $5 \%$ glycerol at $-60^{\circ} \mathrm{C}$.

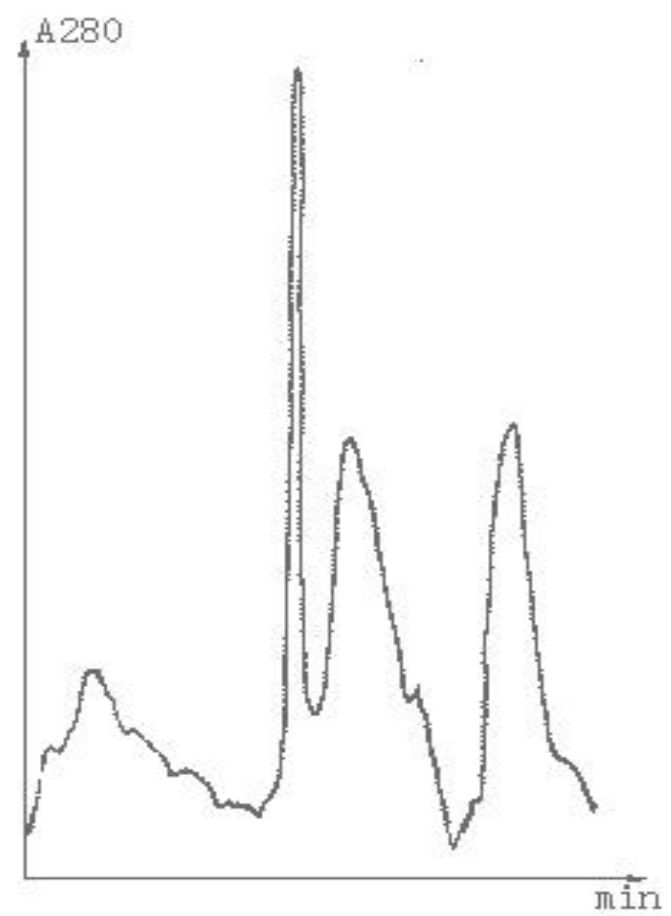

Figure 2. Purification of CAD through a DEAE-Sephorase Cl-6B ionic exchange chromatography 


\section{Reaction medium}

$20 \mathrm{mM}$ Tris- $\mathrm{HCl}$ (pH 7.5) buffer containing $4 \mathrm{mM}$ glutamine (or ), $4 \mathrm{mM}$ aspartate, $50 \mathrm{mM} \mathrm{KCl}, 25 \mathrm{mM}$ $\mathrm{MgCl} 2,15 \mathrm{mM}$ ATP, $4 \mathrm{mM} \mathrm{NaHCO} 3$ and various amount of dimethyl sulphoxide was used as reaction medium.

\section{Dihydroorotate biosynthesis}

$1 \mathrm{~mL}$ of CAD (10mg of protein) and $10 \mathrm{ml}$ reaction medium, vortex-mixing and returning to incubation at $37^{\circ} \mathrm{C}$. After $30 \mathrm{~min}$ the reaction were stopped and the entire contents of the reaction vials were lyophilized and then extracted by ethyl acetate.

\section{RESULTS AND DISCUSSION}

CAD is a mammalian multifunctional polypeptide which catalyzes the first three steps in the de novo pyrimidine biosynthetic pathway. The CAD polypeptide is organized into numerous structural domains that fold autonomously and have distinct functions. One of these domains, the $40 \mathrm{KDa}$ glutaminase domain (GLN), hydrolyzes glutamine and transfers ammonia to the 120 $\mathrm{kDa}$ synthetase domain (CPS) where it is used along with bicarbonate and ATP in the biosynthetic reaction. unusual multiple reaction has been purified in a homogenous state. The CAD is a dimeric enzyme which requires as a cofactor [7-10].

In common with other multifunctional proteins, CAD comprises a number of compact globular domains connected by more exposed polypeptide regions. The product of one step is channeled to the substrate binding site of the enzyme for the next step in the pathway. CAD freely releases the products of the first and second steps of the pathway to the cellular milieu, and the second and third steps of the pathway freely utilize substrates provided from outside the enzyme complex. The multifunctional proteins, CAD, has a molecular weight of about 210,000, forms trimers, hexamers and higher oligomeric forms.

Since metabolic channeling in enzyme complex such as CAD may arise from the close proximity of enzyme active sites, this implies that changes in conformation on binding allosteric effectors or active-site-directed ligands may be transmitted to a neighbouring domain, even one catalyzing a distinct reaction, and may alter the range of proteolytic products.

Enzymes in immobilized form may be deliberately fixed in the vicinity of other enzymes that participate in a given reaction sequence, thereby increasing the overall catalytic efficiency of multistep conversions, because the entropic contribution, arising from the requirement of productive collision of the products of the first reaction with the enzyme catalyzing the second reaction, is dramatically reduced when the reaction becomes intraparticle rather than interparticle. Hence, if two enzymes acting in series are immobilized side by side on a given support matrix, the intermediate product has an extremely high probability of reacting further before it can diffuse out.
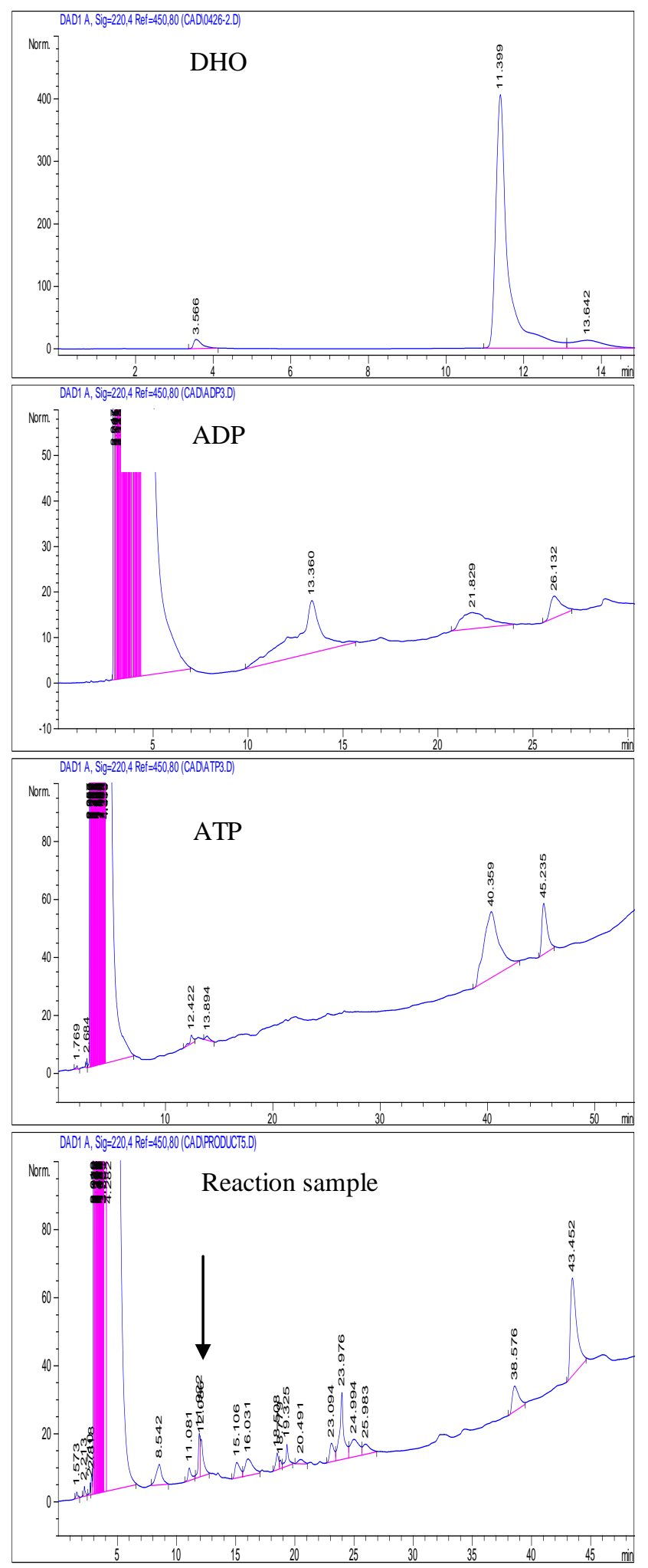

Figure 3. HPLC of DHO, ADP, ATP and reaction sample

The mammalian glutamine-dependent carbamyl phosphate synthetase is unstable in conventional aqueous 
buffers. It can be stabilized by the used of $30 \%(\mathrm{~V} / \mathrm{V})$ dimethyl sulfoxide and 5\% (V/V) glycerol.

The initial studies involving only aqueous system did not appear promising, since under the initial reaction conditions DHO wasn't obtained.

Using aqueous solution as reaction medium, it has been found that the second intermediate in the reaction, carbamoyl-aspartate, is accumulated, but no dihydroootate is produced. (Since carbamoyl aspartate is non-UV absorbing, it was assayed indirectly by measurement 5(acetic acid)-hydantoin. Carbamoyl aspartate was converted to 5-(acetic acid)-hydantoin by cyclization under acidic and heated condition). According to, the conversion of carbamyl-aspartate to dihydroootate is freely reversible. Accumulation of carbamoyl aspartate indicates that under these conditions the equilibrium strongly favors carbamyl-aspartate.

In order to overcome the unfavorable reaction equilibrium, an water-organic system has been used as reaction medium. Since $\mathrm{CAD}$ can be stabilized by dimethyl sulfoxide and glycerol, dimethyl sulfoxide containing Tris- $\mathrm{HCl}$ buffer solution has been selected as reaction medium. When the concentration of aqueous was lower than $5 \%(\mathrm{~V} / \mathrm{V})$, dihydroootate has been found (Figure 3). However, the use of other organic solvent proved disappointing, as CAD was very unstable.

The experimental data obtained is consistent with a model of catalysis where cyclization of CA or ring cleavage of DHO is dependent upon the presence of acidic or basic forms, respectively, of a catalytic residue of the enzyme-substrate complex with a pKa of 7.1.

Study showed that the biosynthetic activity of dihydroorotase is highest below $\mathrm{pH} \mathrm{7,0}$ and decreases rapidly at alkaline $\mathrm{pH}$. The equilibrium between $\mathrm{CA}$ and DHO favors formation of DHO at $\mathrm{pH} 5.8$ while at $\mathrm{pH} 7.0$ formation of CA is favored.

Again the organic phase containing reaction substrates and products eluted in a peak with the tR value anticipated for a pure standard of dihydroorotate, suggesting this metabolite was indeed dihydroorotate. The tR value for dihydroorotate was approximately $11.1 \mathrm{~min}$, The $\mathrm{tR}$ value for ATP being $40.4 \mathrm{~min}$, ADP $13.4 \mathrm{~min}$.
The identity of the peak eluting at $11.1 \mathrm{~min}$ was established by injecting various concentrations of the dihydroorotate onto the Zarbox column. In each instance a single peak with a tR value of $11.1 \mathrm{~min}$, identical to the major peak in the reaction medium..

\section{REFERENCES}

[1] Serre V., Penverne B., Souciet J.-L., Potier S., Guy H., Evans D., Vicart P. and Hervé G., Integrated allosteric regulation in the S. cerevisiae carbamylphosphate synthetase $\quad-$ aspartate transcarbamylase multifunctional protein, BMC Biochemistry 2004, 5:6

[2] Coleman, P.F., Suttle, D.P., and Stark, G.R, Purification from hamster cells of the multifunctional protein that initiates de novo synthesis of pyrimidine nucleotides. J. Biol. Chem. 1977,252,63796385

[3] Juan A. Hermoso, Getting CAD in Shape: The Atomic Structure of Human Dihydroorotase Domain

[4] Grande-García A, Lallous N, Díaz-Tejada C, Ramón-Maiques S, Structure, Functional Characterization, and Evolution of the Dihydroorotase Domain of Human CAD, Structure. 2014,22(2): 185-198

[5] Penverne B, Belkaid M, Herve G: In situ behavior of the pyrimidine pathway enzymes in Saccharomyces cerevisiae. 4 . The channeling of carbamylphosphate to aspartate transcarbamylase and its partition in the pyrimidine and arginine pathways. Arch Biochem Biophys 1994, 309:85-93.

[6] Shoaf W.T. Jones M.E. Uridylic acid synthesis in Ehrlich ascites carcinoma. Properties, subcellular distribution, and nature of enzyme complexes of the six biosynthetic enzymes.Biochemistry, 1973, 12, 4039-4051

[7] Minic Z, Simon V, Penverne B, Gaill F, Hervé G. Contribution of the bacterial endosymbiont to the biosynthesis of pyrimidine nucleotides in the deep-sea tube worm Riftia pachyptila. J Biol Chem. 2001 Jun 29;276(26):23777-23784

[8] Robin JP, Penverne B, Hervé G., Carbamoyl phosphate biosynthesis and partition in pyrimidine and arginine pathways of Escherichia coli. In situ properties of carbamoyl-phosphate synthase, ornithine transcarbamylase and aspartate transcarbamylase in permeabilized cells. Eur J Biochem. 1989 Aug 15;183(3):519-28

[9] Belkaïd M, Penverne B, Hervé G, In situ behavior of the pyrimidine pathway enzymes in Saccharomyces cerevisiae. 3. Catalytic and regulatory properties of carbamylphosphate synthetase: channeling of carbamylphosphate to aspartate transcarbamylase.. Arch Biochem Biophys. 1988 Apr;262(1):17180.

[10] Penverne B, Hervé G,In situ behavior of the pyrimidine pathway enzymes in Saccharomyces cerevisiae. I. Catalytic and regulatory properties of aspartate transcarbamylase., Arch Biochem Biophys. 1983 Sep;225(2):562-575. 